\title{
Extrinsic and intrinsic mechanisms regulating adult mammalian neural stem cells and neurogenesis
}

\author{
Hongjun Song ${ }^{1}$ \\ ${ }^{1}$ Institute for Cell Engineering, Departments of Neurology and Neuroscience, Johns Hopkins University School of Medicine, \\ Baltimore, MD, USA.
}

New neurons are continuously generated from adult neural stem/progenitor cells (NSCs) residing in the subventricular zone of the lateral ventricles and the subgranular zone of the hippocampal dentate gyrus in all mammals examined, including humans. Outside of these two neurogenic regions, proliferating NSCs rarely give rise to functional neurons under physiological conditions. During active adult neurogenesis, NSCs generate functional neurons through orchestrated steps, including cell proliferation, fate specification, neuronal migration, axonal and dendritic growth, and finally synaptic integration into the existing circuitry. As in other somatic stem cell systems, neurogenesis from NSCs in the two neurogenic regions of the adult brain is tightly regulated by the highly specialized microenvironment surrounding the NPCs. These "neurogenic niches" not only anatomically house adult NSCs, but also functionally control their development in vivo. Using multiple approaches for birthdating, genetic marking and manipulation of proliferating NSCs and their progeny in the dentate gyrus of adult mice, we have characterized the sequential events of adult neurogenesis in vivo. Our studies have identified multiple cell types as well as signaling molecules within the unique neurogenic niche of the dentate gyrus that control the coordinated process of adult neurogenesis. Distinct from developing neurogenesis, adult neurogenesis proceeds in the setting of ongoing activity of the mature circuitry and is regulated by many physiological and pathological stimuli. We have identified several factors that are released from different cell types within the local niche in an activity-dependent fashion to either positively or negatively regulate various aspects of adult neurogenesis. In addition these extrinsic factors, we have also identified intrinsic factors that play essential roles in adult hippocampal neurogenesis. We hope a better understanding of cellular and molecular mechanisms of the neurogenic niche may lead to novel strategies for functional neuronal replacement therapy after injury or degenerative neurological disease.

Cell Research (2008) 18:s88. doi: 10.1038/cr.2008.178; published online 4 August 2008

Correspondence: Hongjun Song

E-mail: shongju1@jhmi.edu

Hongjun Song, PhD, Dr Song received his a BS in Biology from Peking University, a MA from Columbia University and a PhD in biology from University of California at San Diego. He is current Associate Professor of Neurology and Neuroscience at Johns Hopkins University
School of Medicine and a member of Neuroregeneration and Repair Program of Institute for Cell Engineering. He has received Klingenstein Fellowship Awards in the Neuroscience, McKnight Scholar Award and Young Investigator Award of the Chinese Biological Investigator Society. Dr Song's research focuses on adult neural stem cells and neurogenesise in mammals. Please see his laboratory website for more details. http:// neuroscience.jhu.edu/HongjunSong.php 